\title{
Orbital Stem Cells
}

\author{
Sara T. Wester
}

Published online: 22 July 2014

(C) Springer Science + Business Media New York 2014

\begin{abstract}
Stem cell research in the field of ophthalmology over the past two decades has advanced knowledge and treatment dramatically. Bone marrow-derived stem cells (BM-MSCs) have been widely studied and have many applications in the eye and other organs; since processed lipoaspirate was first identified as a source of adipose tissue derived stem cells (ASCs), further research has blossomed in this exciting new area. Despite the growth in research involving systemic adipose tissue, the orbital adipose tissue, which is unique in its embryology and has an abundant supply of stem cells, has only recently gained attention. The purpose of this chapter is to outline our current knowledge and recent advances in stem cell research and applications in the orbit, particular orbital adipose stem cells. This chapter will review the fundamentals of adipose stem cell research, the role of orbital fat, dysregulation of orbital adipogenesis, new research on orbital fat stem cells (OFSCs), and potential applications in inflammatory disease and reconstructive and esthetic surgery.
\end{abstract}

Keywords Orbital Adipose Tissue - Adipose Stem Cells . Orbital Fat Stem Cells - Thyroid Eye Disease . Prostaglandin-Associated Periorbitopathy

S. T. Wester $(\bowtie)$

Division of Oculoplastic Surgery, Department of

Ophthalmology, Bascom Palmer Eye Institute, University of Miami Miller School of Medicine, 900 NW 17th St, Miami, FL

33136, USA

e-mail: swester2@med.miami.edu

\section{Introduction}

Stem cells are functionally defined as (a) cells that can selfrenew and provide ongoing populations of identical daughter cells with the same unrestricted proliferation potential and (b) cells that are multipotent and able to give rise to all cell lineages in a particular tissue. Stem cells repopulate damaged or lost tissue either through differentiation into tissue appropriate cells and/or by releasing paracrine signaling molecules to recruit inflammatory cells and other tissue progenitor cells. As such, these cells have a key role in therapeutic tissue regeneration [1]. The initial work on adult stem cells was focused on bone marrowderived stem cells (BM-MSCs) and highlighted the multipotency of these adult stem cells [2]. BM-MSCS offer significant potential for treatment of myriad disease and still are an extremely exciting area of stem cell research. More recently, however, adipose tissue has also been recognized as a potent and easily accessible reservoir of stem cells.

\section{Adipose Stem Cells}

Adipose tissue contains: adipocytes, adipose stem cells (ASCs), vascular endothelial cells, pericytes, and fibroblasts (pre-adipocyte fibroblasts, macrophages, and extracellular matrix). Adipose tissue has been used for many years for restorative and esthetic treatments, with autologous fat soft tissue augmentation dating back to the $19^{\text {th }}$ century [3]. It was not until more recently, however, that processed lipoaspirate (PLA) was shown to contain a reservoir of viable stem cells with mesodermal and ectodermal capacity, suggesting a new source of adult stem cells the adipose tissue [4]. Since then, numerous data have shown that ASCS have an almost identical transcriptional profile for genes related to stem cell phenotype as BM- 
MSCs [5]. In addition, the expression of various stem cell markers has been observed to be similar with ASCs to the well-documented BM-MSC profile [6, 7]. ASCs have also been shown to exhibit fibroblast-like morphology and multipotency toward adipogenic, osteogenic, and chondrogenic differentiation [8].

There are several interesting and important differentiating factors between ASCs and BM-MSCs. Adipose tissue generates a much higher yield of stem cells than BM-MSCs [9]. In addition, the ease of harvest makes this tissue easily accessible with minimal downtime. ASCs also serve a role in tissue regeneration, secreting potent growth factors (VEGF, FGF-2, IGF-1, and HGF) [10]. Several studies have shown that ASCs can play a critical role in wound repair, with improved healing and graft survival. In this context, the fact that these cells lack immunogenicity [11] and play an immunomodulatory role [11] is extremely beneficial. In addition, significant phenotypic heterogeneity has been demonstrated among ASCs, with variations in differentiation patterns based on receptor positivity [12]. The CD105 subset has been shown to have a high chondrogenic potential [13], and the p75 neurotrophin receptorpositive ASCs have been shown to have higher potential for adipogenesis, osteogenesis and potential to differentiate into neuronal cells $[14,15]$. The ASC subpopulation with CD31-/CD34 + markers has been shown to have the highest potential for adipogenesis among this heterogeneous population of stem cells [16]. In addition, it has been shown that there is a subset of ASCS which derive from the neural crest and that neural crest cells, in the appropriate environment, can differentiate into adipocytes [17]. The phenotype of these neural crest derived "adipocyterestricted" progenitors has been shown to be distinct from non-neural crest derivatives in the ASC population [12]. The unique diversity of the adipose tissue, therefore, may enable a broad spectrum of applications of these ASCs across myriad pathological conditions. In order to understand the potentil role of orbital ASCs, we first need to review the role of orbital fat.

\section{Role of Orbital Fat-the Good, the Bad, the Ugly}

What is the Role of Orbital Fat?

Orbital fat serves as an adipose cushion for the eye, through which important vessels, nerves and extraocular muscles pass. This soft cushion allows the extraocular muscles to slide easily for movement of the eye. It lines the bony orbit, which provides the support framework for the eye, and within which lies the eye, optic nerve, extraocular muscles, nerves, fat, lacrimal gland, and blood vessels (see Figure 1). The orbital fat also provides volume to the orbital

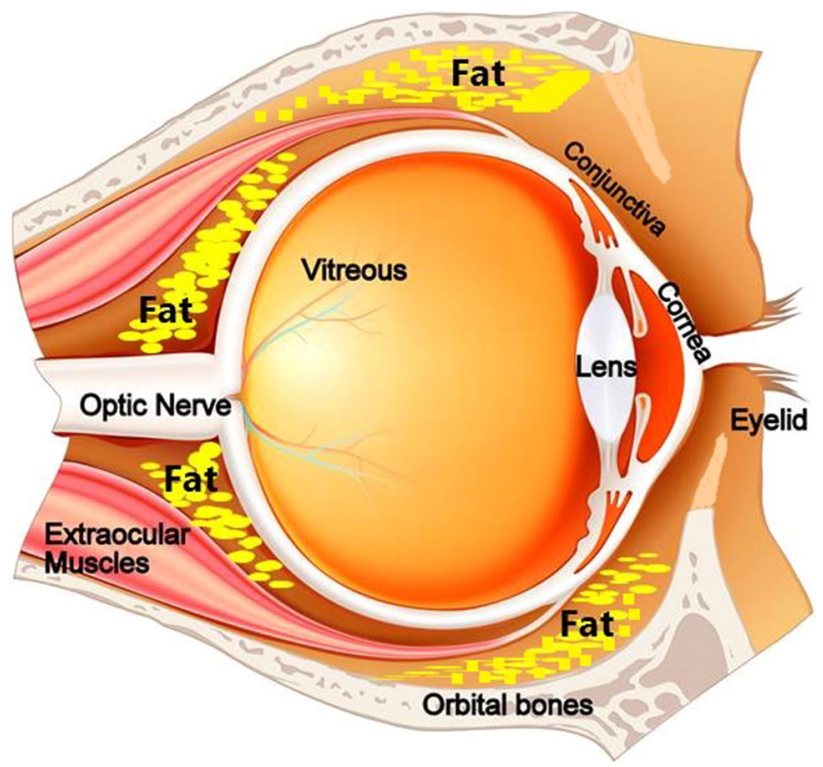

Fig. 1 Anatomy of the orbit (Illustration by Jennifer Thomson, PhD)

cavity. As such, any process that leads to expansion of the orbital fat compartment (such as can occur with Thyroid eye disease), or loss of orbital fat (such as in periorbitopathy), can have significant consequences for the integral structures which lie within. Loss of orbital fat can lead to a sunken appearance of the eye, and in severe cases may actually disrupt extraocular motility. Expansion of the orbital fat with de novo adipogenesis as seen in thyroid eye disease can compress the structures that traverse this normally semifluid cushion and lead to vascular or neurologic complications of the eye, even blindness.

An understanding of the stem cell population within this unique bony cavity has already provided numerous exciting potential therapeutic applications throughout the body. We must look at this stem cell population, however, to better understand the diseases that affect the delicate balance of this bony cavity.

\section{Dysregulation of Orbital Fat: What Happens to Orbital Fat in Pathological States?}

Adipogenesis is a complex process through which a variety of genes become induced leading to lipoprotein lipolysis (cellular uptake of fatty acids and synthesis of fatty acids and triglycerides [18, 19]. Leptin is a gene expressed in cells during late differentiation into adipocytes and can be a useful marker of adipocyte differentiation [20, 21]. Fatty acid-binding protein, fatty acid synthase, complement factor $\mathrm{D}$, adiponectin, and leptin are all adipogenic markers which are used in the analysis of adipose tissue. In 
addition, we know that upregulation of peroxisome proliferator-activated receptor gamma (PPAR $\lambda$ ) indicates an adipogenic fate commitment and inhibition of PPAR $\lambda$ blocks the differentiation of orbital fibroblasts into adipocytes and thus downregulates adipogenesis [22].

\section{Upregulation of Adipogenesis-Thyroid Eye Disease}

Thyroid eye disease is an autoimmune inflammatory disease that has significant orbital consequences. Clinical signs of thyroid eye disease can vary from mild to severe and include proptosis (bulging of the eye), strabismus (double vision), bulging eyelids, exposure keratopathy, eyelid retraction, and optic neuropathy. Enlargement of the extraocular muscles and expansion of the orbital fat from stimulation of orbital fibroblasts and adipogenesis in the fixed volume of the bony orbit can lead to these symptoms. The pathology of thyroid eye disease involves an autoantibody cross-reaction from the thyroid-stimulating hormone (TSH) receptor with orbital fibroblasts. This leads to orbital fibroblast stimulation of lymphocytes, release of various cytokines and production of glycosaminoglycans that increase volume by osmotic edema. Fibroblast proliferation and infiltration into muscle fibers leads to extraocular muscle fibrosis and disabling diplopia [23]. In addition, preadipocyte fibroblasts in this environment can differentiate into adipocytes, leading to expansion of the orbital fat cavity.

De novo adipogenesis within orbital tissues is an important factor in the pathology of TED [24]. Orbital TSHr expression has been detected in patients with TED [25] and the low-level surface TSHr displayed on orbital fibroblasts increases following differentiation in vitro into adipocytes [26•]. We know that the autoimmune process of Graves' disease (GD) involves activating Abs against TSHR. Proliferation of fibroblasts and adipocytes and secondary hyaluronan accumulation (among other aminoglycosides) can lead to tissue volume expansion, orbital congestion, exophthalmos, and compressive optic neuropathy [27]. However, it has been shown that the orbital adipose tissue expansion that occurs in thyroid eye disease is not likely due to edema alone. The density of orbital fat (which is a function of the state of hydration) in neuroimaging studies of TED patients is similar to that of normal orbital fat [28]. In addition, the weight and volume of the orbital adipose tissue in TED patients were found to be increased compared to normal tissues (with the degree of increase correlated with the degree of exophthalmos [29]. As opposed to the accumulation of glycosaminoglycans that occurs in the extraocular muscles and leads to edema, therefore, the process of orbital adipose tissue changes has been thought to be likely due to increased adipogenesis. This theory has more recently been proven on a molecular basis. The expression of various proteins associated with adipogenesis (such as leptin) in human orbital preadipocyte fibroblasts (using immunohistochemistry) was found to be 17-36 times greater in those cultures that had differentiated into adipocytes with a functional thyroid-stimulating hormone receptor than controls on day 7 , suggesting higher amount of newly differentiated adipocytes and "de novo adipogenesis" occurs. [30].

In addition, circulating fibrocytes are overabundant in GD and can infiltrate the orbit and thyroid $(16,17)$. Fibrocytes are bone marrow-derived progenitor cells that can express an array of surface markers and can differentiate into fat cells [31]. Interestingly, orbital fibroblasts are typically CD34-, but it has been shown that CD34 + fibrocytes are present in much higher quantities in patient with GD compared to healthy controls [32]. It would therefore appear that $\mathrm{CD} 34+$ orbital fibroblasts could derive from circulating fibrocytes (circulating fibrocytes are involved in the inflammatory cascade as they are involved in antigen presentation, tissue remodeling, wound healing, and fibrosis) [33, 34] and have been shown to increase cytokine production in TED patients [35]. In addition, Thyroglobulin and TSHR mRNA expression is seen in CD34 + orbital fibroblasts of GD patients, but not in CD34- fibroblasts of these same patients, implicating fibrocytes in the inflammatory cascade in TED [36]. If these bone marrow-derived progenitor cells play a significant role in TED through local fibrocyte biosynthetic activity [36], a better understanding of the orbital adipose stem cell population in thyroid patients compared to controls will have significant clinical implications. In addition, OFSCs have unique expression levels of adipohormones depending on the stage of differentiation, with low baseline levels of adiponectin becoming extremely high in OFSCdifferentiated cells [37]. Given the role of adiponectin in inhibiting inflammation [37], this also may also have a key role in TED.

\section{Downregulation of Adipogenesis-Prostaglandin- Associated Periorbitopathy}

On the other end of the spectrum, various inhibitors of the angiogenesis pathway can lead to toxicity induced necrosis of adipose tissue or fat atrophy from nonspecific inflammatory responses [22]. It is well known that prostaglandin analogs may have side effects on the surrounding tissues when used in the treatment of glaucoma [38]. PGF2a has been shown in myriad studies to have an inhibitory effect on adipocyte differentiation [39-41] and block adipogenesis [42]. Prostaglandin analogs have been found to lead to a decrease in the orbital adipose population, via inhibition of human preadipocyte differentiation and intracellular lipid accumulation [22]. Increased fat density, reflective of 
a lower mean number of adipocytes per unit area has also been shown after treatment with prostaglandin analogs [42]. On a cellular level, PGF2a and topical prostaglandin analogs down regulate the expression of various transcription factors necessary for adipogenesis [22], confirmed with orbital adipocytes exposed ex vivo to prostaglandin analogs [43]. Prostaglandin analogs also inhibit the accumulation of intra-cytoplasmic lipid droplets and have demonstrated decreased expression of lipoprotein lipase [22]. If we remember back to the adipogenesis pathway previously described, PPAR $\lambda$ plays a key role in adipogenesis. Others have also shown that adipocytes in the presence of PGF2 $\alpha$ agonists have decreased expression of adipocyte markers as well [44]. It has been shown that topical prostaglandin analogs may therefore lead to periorbital fat atrophy through the above mentioned mechanisms [22]. This has been well documented in the clinical setting with retrospective studies and case series; we are currently conducting a prospective observational study to analyze the time of onset and prevalence of this side effect. Given what we know about the dysregulation of the orbital adipose compartment seen in prostaglandin-associated periorbitopathy, we can use this data to further our understanding of changes in the OFSCs and potentially to address diseases of increased adipogenesis such as TED.

\section{What are Orbital Adipose Stem Cells? What is Their Embryogenesis? Why are Orbital Stem Cells Unique?}

The ease of access to orbital adipose tissue during routine eyelid surgery makes it an interesting target for analyzing the orbital fat stem cells (OFSCs), hence the recent proliferation of research in this area. Data have shown a similar growth kinetic between orbital fat derived stem cells (OFSCS) and BM-MSCS [45], suggesting this adipose population has many of the same characteristics as ASCs. In addition, there is significant overlap of surface markers between the two [17, 19, 20]. Interestingly, some studies have shown OFSCs to be negative for hematopoietic stem cell markers (CD34, CD133) and endothelial progenitor cell marker CD31 suggesting that these cells were not of hematopoietic origin [37], while others have shown CD34 positivity [46••]. In addition, under osteogenic, chondrogenic and adipogenic induction, these cells have shown differentiation ability into osteoblasts, mature chondrocytes, and adipocytes [37] [45].

Interestingly, orbital adipocytes (which are continuous with the nasal fat pad) are derived from neural crest cells, versus adipose tissue throughout the body, which is generally mesodermal in origin [47]. As previously highlighted, this unique subpopulation of ASCs has unique differentiation characteristics as well [17]. The central preaponeurotic fat is thought to be similar to systemic adipose tissue and is in large part derived from mesoderm. Both nasal and central adipose depots have been found to have the potential to differentiate into adipocytes, smooth muscle, and neuronal/ glial lineages [46••], but their unique embryology may play an interesting role in their potential applications. For example, when further breaking up OFSCs into nasal and central components, the nasal adipose stem cells express a higher level of antigens (such as CD34) correlated with neurogenic differentiation potential [46*0]. Research has suggested, however, that the pathway for differentiation is modulated at several levels [46*0]. Tracing back the embryology of the eye and orbital tissue, we remember that a large component of ocular and orbital contents derive from neuroectoderm. The corneal stroma is derived from neural crest, similar to the nasal fat pads/orbital fat. Interestingly, therefore, orbital fat and the corneal stroma have similar embryogenesis. Due to the similar embryonic origin of the orbital connective tissue and orbital adipose tissue with many other ocular tissues, this adipose tissue can be a great source of stem cells for ocular disease (i.e., cornea) $[37,45]$. This has been shown when comparing OFSCs to ASCs, with the percentage of epithelial-specific antigen (ESA)-positive cells significantly increased in OFSCs only after a 5-day mix culture with human corneal epithelial cells suggesting significant mesenchymal to epithelial shifting occurred in OFSCs but not in ASCs [37]. In addition, ZO-1 (a marker of epithelial tight junction) became detectable in OFSCs, demonstrating the potential of these stem cells to differentiate into epithelial cells [37]. In the presence of HCE-T cells, OFSCs also expressed CK19 (corneal epithelial progenitor marker) and CK3 (mature $\mathrm{K}$ epithelial cell marker), and ASCs did not express these markers in the same conditions [37]. The potential applications of OFSCs to treat ocular surface disease are currently being studied and have significant potential.

\section{What are the ideal ways to isolate OFSCs?}

We have recently analyzed ways in which to improve upon traditional adipose stem cell isolation techniques using OFSCs. Preliminary data from our research suggest that the OFSCs have not been well isolated by the conventional method (unpublished data). The stromal vascular fraction (SVF), a heterogeneous mixture of cells isolated by digestion of adipose tissue with collagenase and filtering off of the remaining cells, has been shown to contain multipotent cells, which are referred to as adipose derived stem/stromal/progenitor cells (ASCs) [48••, 49]. It has been shown that these cells can differentiate into multiple phenotypes, including adipose, muscle, bone, neuronal, endothelial, hepatocyte, and epithelial-like cells [48••, 50-53]. The remaining cells are typically discarded and little data 
exists on the potential isolation of stem cells from this tissue. Our initial data suggest that the remaining cell (RC) fraction contains progenitors better than the stromal vascular fraction (SVF), which is the traditional assumption for the location of ASCs (unpublished data). This has significance not only for analysis of the orbital adipose tissue, but also for the use of adipose stem cells in soft tissue augmentation. Whether for functional or cosmetic deficits, the "stem-ness" of the cells used are essential to the long-term benefits possible in treatment of disease, as discussed below. In addition, given the phenotypic heterogeneity of ASCs, isolating each stem cell population is essential to maximize their potential applications.

\section{What are the Current Potential Applications of Orbital Adipose Stem Cells?}

\author{
Applications of ASCs/OFSCs in Pathology Affecting \\ the Orbit and Ocular Tissue
}

As described above, the pathogenesis of thyroid disease involves expansion of extraocular muscles, fibrosis, and dysregulation of adipogenesis. Given the role of de novo adipogenesis in thyroid eye disease, we hope to understand the shifts in stem cell markers that occur in thyroid eye disease patients. As has been well documented, traditional treatment of TED focuses on the ocular sequelae and does not reverse or halt the disease process. In cases of compressive optic neuropathy, steroids (IV or oral) are used as treatment in some cases, but the side effect profile is notable. As such, treatment advances have more recently been focused on alternative forms of immunomodulation [54]. Severe compressive optic neuropathy often requires orbital decompression surgery to prevent permanent blindness. It is important to note that the ophthalmopathy may be progressive during times of thyroid dysfunction making surgical intervention more risky. In addition, several surgical interventions may be required as patients that undergo orbital decompression may also eventually need strabismus surgery for diplopia followed by surgery for eyelid retraction. While these surgical interventions are often successful, they treat the end result of disease as opposed to reversing or halting the disease process and are not without risk. Given these issues, research is focused on treating patients prior to the development of severe orbital disease, to halt the disease process at the molecular level.

We know that both normal and TED patients have orbital preadipocytes, derived from mesenchymal stem cells and that this population likely plays a role in the orbital inflammation seen in TED [55]. Recent data have shown the role of fibrocytes in thyroid eye disease, as highlighted above. CD40 levels on fibrocytes and orbital fibroblasts in TED patients have been shown to be significantly upregulated, via IFN- $\lambda$ upregulation at the pretranslational level [56]. In addition, $\mathrm{CD} 45+\mathrm{CD} 34+\mathrm{Col} 1+$ fibrocytes have been found to be increased in the serum of patients with TED [56], but this has not been demonstrated in the orbital adipose tissue itself. It has also been shown that fibrocyte-to-adipocyte differentiation is drive by the peroxisome proliferator-activated receptor (PPAR) agonist troglitazone [57]. Understanding the unique stem cell population of OFSCs and the phenotypic variability may provide insight into the processes that regulate this transition in vivo in the orbital adipose tissue compartment. In addition, it has been shown that transforming growth factor (TGF) $\beta 1$ leads to fibrocyte-to-myofibroblast differentiation through various pathways [31], and this could potentially contribute to the extraocular muscle involvement of some TED patients.

Thus, an understanding of the in vivo changes in the OFSC of thyroid patients may provide significant insight and allow targeted therapy that would circumvent the need for multiple surgeries or anti-inflammatory medications. We have been analyzing the variations in the orbital adipose stem cell population of patients with thyroid disease, and have found upregulation in certain key markers that may provide opportunities for molecularly-based therapy (unpublished data), and are supported by previous data from systemic serum analysis [35, 58]. It is possible as well, that an understanding of the pathologic and physiologic variations in the OFSCs may offer insight into local therapeutic options for patients with thyroid eye disease.

In addition, as previously highlighted, the similar embryonic derivation of certain ocular tissue and orbital adipose tissue may allow for improved treatment of ocular surface disease. In a mouse model of cornea alkali burns, OFSCs where found to enhance healing through the inhibition of inflammation and corneal epithelial differentiation by OFSCs (in the early stage of healing) [45]. In the late stage, stromal engraftment of OFSCS was thought to enhance corneal transparency [45]. Other data have shown the potential applications of OFSCs in treating ocular disease [37]. Burn patients often have significant tissue loss and may suffer not only from ocular surface sequelae, but also loss of periocular tissue. Given what we know about OFSCs, these stem cells may provide exciting new treatment options for these patients, reducing inflammation and modulating the wound healing process. In the periocular tissue, patients with a history of radiation for cutaneous malignancies may also have poor wound healing. In addition, patients with radiation damage may also have decreased functionality of their own stem cells. Certain factors integral to attracting stem cells to the wound, thus essential to the wound healing process, can also become deficient with age and diabetes [59]. Because of the deficiency in stem cell functionality in cases of radiation, 
diabetes, and age, stem cell application may play a key role in wound healing in these cases.

\section{Applications of ASCs in Wound Healing}

Given what we know about ASCs and their potential to aid in wound healing, we may be able to use OFSCs to accelerate wound healing through various pathways. ASCs activate dermal fibroblasts and keratinocytes via paracrine mechanisms [60]. In wound repair models, stem cells migrate to the site of injured tissue, support the proliferation and differentiation of progenitor cells, replace lost cells via differentiation, increase tissue angiogenesis, secrete growth factors and thus play a role in matrix remodeling, and activate immune cells [61]. Several animal and human models have shown the benefit of ASCs in wound healing in vivo. ASCs can help regenerate dermis in a porcine model when seeded onto large collagen scaffolds [62]. In addition, ASCs have shown improved wound healing in humans when applied in sheets placed on the wound bed with improved healing seen in 18 out of 23 patients, and growth factor and cytokine production that were thought to stimulate the wound milieu and lead to an ongoing "edge effect" [63]. Other studies have shown an increased survival area in ischemic skin flaps [64]. Improved wound healing in non-healing wounds with topical application $[65,66]$ and improvement in radiationrelated wounds and ulcers [67] has also been demonstrated. Interestingly, the stromal vascular fraction of ASCs has been found to lack the endothelial marker CD31, suggesting a difference in this stem cell population. Despite these differences, these cells were still able to differentiate into endothelial cells and promote angiogenesis [51] and thus have a significant potential role in ischemic tissue.

Interestingly, OFSCs have been studied in wound healing as well. The application in corneal burn models was elucidated previously [45]. Acute lung injury is another pathological state in which human OFSCs were shown to inhibit the acute inflammatory responses, as demonstrated by a decrease in total protein concentration and neutrophil counts, reduced endothelial and alveolar epithelial permeability and decreased neutrophil (Ly6Gexpressing cells) and macrophage (CD68-expressing cells) infiltration, with an associated decrease in systemic proinflammatory chemokines [68]. Extrapolating from preliminary data from OFSCs and ASCs, one could deduce that OFSCs will likely serve a role in assisting with wound healing in ocular surface disease and periocular wounds.

\section{Applications of ASCs in Esthetic Surgery}

One of the key areas in which adipose stem cells have gained significantly popularity is in the cosmetic surgery arena. As mentioned previously, adipose tissue has several key benefits: biocompatibility, no hypersensitivity, low infection risk, abundance, low cost, and natural consistency with good volume augmentation [69•]. Fat transfer, which has been used extensively for esthetics, was first introduced by Neuber in 1893 [3] but was plagued with several issues including the progressive loss of transplanted adipocytes, conversion of the fat to fibrous tissue and poor vascularity leading to cell death.

The technique of harvesting and injection has been revised significantly since then to minimize trauma to the fat cells (delicate aspiration and microinjection of small particles of fat in multiple layers); despite these advances, retention rates of fat vary significantly due to partial necrosis [70]. Given these drawbacks of fat transfer, enhancing the fat with stem cells has been proposed to improve maintenance of the volume of fat injected, enhance angiogenesis and minimize inflammation [70]. Cell-assisted lipotransfer (CAL) is a technique by which ASCs are isolated by enzymatic digestion with collagenase, then filtered and centrifuged and the stromal vascular fraction (SVF), which are thought by many to be the ASC reservoir, is isolated [71•]. The goal of using ASCs with fat injections was to minimize the issues seen with fat transfer of partial necrosis, volume loss, and internal calcification [72].

The goal of highly marketed procedures such as the "Stem cell facelift", therefore, was to restore volume while adding stem cells. In techniques such as this, fat is typically obtained from the lower abdomen via Liposuction, and the stem cells are then isolated via a variety of techniques and injected directly or via implantation with a delivery matrix. As with any technology, there are risks associated with this, and there have been case reports of complications such as bone growth at the site of injection (thought to be due to the interaction of the stem cells with a calcium hydroxyapatite filler which was injected at the same time) [73]. In addition, there are regulatory issues due to the FDA regulations on minimally manipulation for structural tissue (FDA Rule 361).

Adipose stem cells have also been shown to have possible antiwrinkle effects, with wrinkle reduction seen when these cells were injected into the dermis and increased dermal thickness and collagen expression seen on histopathology. [74] These stem cells have also been shown to protect dermal fibroblasts from oxidative stress induced by chemicals and UVB radiation, with reductions in UVB induced apoptosis and improved collagen synthesis of dermal fibroblasts [74, 75]. An improvement in skin texture following fat grafting to the face and in the athymic mouse model has also been seen, with a thickened dermis after grafting and increased synthesis of collagen I fibers [76].

New techniques have sought to evaluate combination therapy using ASCs. For example, fractionated laser has been 
shown to be a potential transdermal delivery mechanism for ASCs [77], with ASCs delivered transdermally through skin pretreated with a laser. Use of this and other laser modalities may enhance the potential wound healing and esthetic applications of ASCs. Recent studies have also looked at whether botulinum toxin (BoNTA) could be helpful with fat graft survival due to tissue stabilization. The cellular integrity of the transplanted fat was found to be higher in those cases with botulinum toxin, which was thought to be due to decreased muscle contractions of the surrounding tissue [78].

Given the similar attributes of OFSCs and ASCs, it is likely the above findings will also apply to OFSCs. In the future, OFSCs may be isolated from orbital fat removed during blepharoplasty and used to improve wound healing, enhance wrinkle reduction and provide volume enhancement in the periocular tissue.

\section{Conclusion}

Orbital stem cells have been studied much less extensively than those in other areas of ophthalmology. The potential clinical applications in this area are broad. Further research into these progenitor cells may provide insight on pathological processes affecting the orbit, such as TED and prostaglandin-associated periorbitopathy, and hopefully enable earlier, preventative treatment. We are currently studying OFSCs in TED and prostaglandin patients to ascertain potential-targeted therapy for TED. In addition, OFSCs, of which the nasal fat pad derives from neural crest cells, may have unique capabilities in terms of treatment of ocular surface disease and burns. Orbital adipose tissue and the reservoirs of stem cells which are routinely excised during blepharoplasty surgery may offer potential treatment for inflammatory disease of the orbit, periocular and ocular wound healing and periocular and facial esthetics.

Conflict of interest Sara Wester has not reported any conflicts of interest.

Human and Animal Rights and Informed Consent This article does not contain any studies with human or animal subjects performed by any of the authors.

\section{References}

Papers of particular interest, published recently, have been highlighted as:

- Of importance

•- Of major importance

1. Lau K, et al. Exploring the role of stem cells in cutaneous wound healing. Exp Dermatol. 2009;18(11):921-33.
2. Pittenger MF, et al. Multilineage potential of adult human mesenchymal stem cells. Science. 1999;284(5411):143-7.

3. Nueber G. Über die Wiederanheilung vollständig vom Körper getrennter, die ganze Fettschicht enthaltender Hautstücke. Zbl F Chirurgie. 1893;30:16.

4. Zuk PA, et al. Human adipose tissue is a source of multipotent stem cells. Mol Biol Cell. 2002;13(12):4279-95.

5. Peroni D, et al. Stem molecular signature of adipose-derived stromal cells. Exp Cell Res. 2008;314(3):603-15.

6. Lee JH, Kemp DM. Human adipose-derived stem cells display myogenic potential and perturbed function in hypoxic conditions. Biochem Biophys Res Commun. 2006;341(3):882-8.

7. Lee $\mathrm{RH}$, et al. Characterization and expression analysis of mesenchymal stem cells from human bone marrow and adipose tissue. Cell Physiol Biochem. 2004;14(4-6):311-24.

8. Mizuno H. Adipose-derived stem cells for tissue repair and regeneration: ten years of research and a literature review. J Nihon Med Sch. 2009;76(2):56-66.

9. Zhu Y, et al. Adipose-derived stem cell: a better stem cell than BMSC. Cell Biochem Funct. 2008;26(6):664-75.

10. Planat-Benard V, et al. Plasticity of human adipose lineage cells toward endothelial cells - Physiological and therapeutic perspectives. Circulation. 2004;109(5):656-63.

11. Chien MH, et al. Systemic human orbital fat-derived stem/stromal cell transplantation ameliorates acute inflammation in lipopolysaccharide-induced acute lung injury. Crit Care Med. 2012;40(4):1245-53.

12. Sowa $Y$, et al. Adipose stromal cells contain phenotypically distinct adipogenic progenitors derived from neural crest. PLoS ONE. 2013;8(12):e84206.

13. Jiang $\mathrm{T}$, et al. Potent in vitro chondrogenesis of CD105 enriched human adipose-derived stem cells. Biomaterials. 2010;31(13):3564-71.

14. Yamamoto $\mathrm{N}$, et al. Isolation of multipotent stem cells from mouse adipose tissue. J Dermatol Sci. 2007;48(1):43-52.

15. Rada T, Reis RL, Gomes ME. Distinct stem cells subpopulations isolated from human adipose tissue exhibit different chondrogenic and osteogenic differentiation potential. Stem Cell Rev. 2011;7(1):64-76.

16. Li H, et al. Adipogenic potential of adipose stem cell subpopulations. Plast Reconstr Surg. 2011;128(3):663-72.

17. Billon N, et al. The generation of adipocytes by the neural crest. Development. 2007;134(12):2283-92.

18. Sorisky A. From preadipocyte to adipocyte: differentiationdirected signals of insulin from the cell surface to the nucleus. Crit Rev Clin Lab Sci. 1999;36(1):1-34.

19. Smas CM, Sul HS. Control of adipocyte differentiation. Biochem J. 1995;309(Pt 3):697-710.

20. Mitchell SE, et al. ob gene expression and secretion of leptin following differentiation of rat preadipocytes to adipocytes in primary culture. Biochem Biophys Res Commun. 1997;230(2):360-4.

21. Leroy P, et al. Expression of ob gene in adipose cells. Regulation by insulin. J Biol Chem. 1996;271(5):2365-8.

22. Choi, H.Y., et al., In vitro study of antiadipogenic profile of latanoprost, travoprost, bimatoprost, and tafluprost in human orbital preadiopocytes. J Ocul Pharmacol Ther. 28(2): p. 146-52.

23. Hiromatsu Y, et al. Cytokine profiles in eye muscle tissue and orbital fat tissue from patients with thyroid-associated ophthalmopathy. J Clin Endocrinol Metab. 2000;85(3):1194-9.

24. Kumar S, et al. Evidence for enhanced adipogenesis in the orbits of patients with Graves' ophthalmopathy. J Clin Endocrinol Metab. 2004;89(2):930-5.

25. Feliciello A, et al. Expression of thyrotropin-receptor mRNA in healthy and Graves' disease retro-orbital tissue. Lancet. 1993; 342(8867):337-8.

26. - Valyasevi RW, et al. Differentiation of human orbital preadipocyte fibroblasts induces expression of functional thyrotropin 
receptor. J Clin Endocrinol Metab. 1999;84(7):2557-62. This study analyzed the role of the TSH receptor (TSHr) antigen in the pathogenesis of Graves' ophthalmopathy $(G O)$. They demonstrated relatively greater $\mathrm{TSHr}$ gene expression in GO than in normal orbital tissue specimens. indicating that orbital preadipocyte fibroblasts increase their TSHr expression with differentiation and suggest that these cells play an important role in the pathogenesis of $G O$.

27. Kahaly G, Forster G, Hansen C. Glycosaminoglycans in thyroid eye disease. Thyroid. 1998;8(5):429-32.

28. Peyster RG, et al. Exophthalmos caused by excessive fat: CT volumetric analysis and differential diagnosis. AJR Am J Roentgenol. 1986;146(3):459-64.

29. Rundle FF, Finlay-Jones LR, Noad KB. Malignant exophthalmos: a quantitative analysis of the orbital tissues. Australas Ann Med. 1953;2(2):128-35.

30. Erickson DZ, et al. Induction of leptin expression in orbital preadipocyte fibroblasts. Thyroid. 2001;11(3):221-6.

31. Hong KM, et al. Differentiation of human circulating fibrocytes as mediated by transforming growth factor-beta and peroxisome proliferator-activated receptor gamma. J Biol Chem. 2007; 282(31):22910-20.

32. Douglas RS, et al. Increased Generation of Fibrocytes in ThyroidAssociated Ophthalmopathy. J Clin Endocrinol Metab. 2010; 95(1):430-8.

33. Bucala R, et al. Circulating Fibrocytes Define a New Leukocyte Subpopulation That Mediates Tissue-Repair. Mol Med. 1994; 1(1):71-81.

34. Pilling, D., et al., Identification of Markers that Distinguish Monocyte-Derived Fibrocytes from Monocytes, Macrophages, and Fibroblasts. Plos One, 2009. 4(10).

35. Gillespie EF, et al. Interleukin-6 Production in CD40-Engaged Fibrocytes in Thyroid-Associated Ophthalmopathy: Involvement of Akt and NF-kappa B. Invest Ophthalmol Vis Sci. 2012;53(12): 7746-53.

36. Fernando R, et al. Human fibrocytes coexpress thyroglobulin and thyrotropin receptor. Proc Natl Acad Sci USA. 2012;109(19):7427-32.

37. Ho JH, et al. Isolation and characterization of multi-potent stem cells from human orbital fat tissues. Tissue Eng Part A. 2011;17(1-2):255-66.

38. Ishida $\mathrm{N}$, et al. Prostanoids in the therapy of glaucoma. Cardiovasc Drug Rev. 2006;24(1):1-10.

39. Serrero G, Lepak N. Endocrine and paracrine negative regulators of adipose differentiation. Int J Obes Relat Metab Disord. 1996; 20(Suppl 3):S58-64.

40. Serrero G, Lepak NM. Prostaglandin F2alpha receptor (FP receptor) agonists are potent adipose differentiation inhibitors for primary culture of adipocyte precursors in defined medium. Biochem Biophys Res Commun. 1997;233(1):200-2.

41. Reginato MJ, et al. Prostaglandins promote and block adipogenesis through opposing effects on peroxisome proliferatoractivated receptor gamma. J Biol Chem. 1998;273(4):1855-8.

42. Park, J., H.K. Cho, and J.I. Moon, Changes to upper eyelid orbital fat from use of topical bimatoprost, travoprost, and latanoprost. Jpn J Ophthalmol. 55(1): p. 22-7.

43. Wladis E, L.H., O'Malley M, Avram D, Cell-signaling effects of prostaglandin analogues on orbital adipocytes. AAO 2012 Subspecialty Day Oculofacial Plastic Surgery; Free Papers - The Best of ASOPRS, 2012: p. 1.

44. Serrero G, Lepak NM. Prostaglandin F-2 alpha receptor (FP receptor) agonists are potent adipose differentiation inhibitors for primary culture of adipocyte precursors in defined medium. Biochemical and Biophysical Research Communications. 1997;233(1):200-2.

45. Lin, K.J., et al., Topical administration of orbital fat-derived stem cells promotes corneal tissue regeneration. Stem Cell Research \& Therapy, 2013. 4.
46. •• Korn BS, Kikkawa DO, Hicok KC. Identification and characterization of adult stem cells from human orbital adipose tissue. Ophthal Plast Reconstr Surg. 2009;25(1):27-32. This study isolated pluripotential adipose-derived stem cells from human orbital adipose during routine blepharoplasty surgery and demonstrated that OFSCs from the nasal and central adipose depots showed the potential to differentiate into the adipocyte, smooth muscle, and neuronal/glial lineages and expressed a CD marker protein profile consistent with that observed for adipose-derived stem cells from other adipose depots.

47. Johnston MC, et al. Origins of avian ocular and periocular tissues. Exp Eye Res. 1979;29(1):27-43.

48. $\bullet$ Zuk PA, et al. Multilineage cells from human adipose tissue: implications for cell-based therapies. Tissue Eng. 2001;7(2):211-28. This was the first study to demonstrate that a population of stem cells could be isolated from human adipose tissue using human adipose tissue, obtained by suction-assisted lipectomy (i.e., liposuction) to yield processed lipoaspirate (PLA) which could be maintained in vitro for extended periods. They demonstrated that PLA cells differentiate in vitro into adipogenic, chondrogenic, myogenic, and osteogenic cells in the presence of lineage-specific induction factors.

49. Rodriguez AM, et al. The human adipose tissue is a source of multipotent stem cells. Biochimie. 2005;87(1):125-8.

50. Brzoska M, et al. Epithelial differentiation of human adipose tissue-derived adult stem cells. Biochem Biophys Res Commun. 2005;330(1):142-50.

51. Planat-Benard V, et al. Plasticity of human adipose lineage cells toward endothelial cells: physiological and therapeutic perspectives. Circulation. 2004;109(5):656-63.

52. Romanov YA, et al. Mesenchymal stem cells from human bone marrow and adipose tissue: isolation, characterization, and differentiation potentialities. Bull Exp Biol Med. 2005;140(1):138-43.

53. Sgodda M, et al. Hepatocyte differentiation of mesenchymal stem cells from rat peritoneal adipose tissue in vitro and in vivo. Exp Cell Res. 2007;313(13):2875-86.

54. Salvi M, et al. Efficacy of rituximab treatment for thyroid-associated ophthalmopathy as a result of intraorbital B-cell depletion in one patient unresponsive to steroid immunosuppression. Eur J Endocrinol. 2006;154(4):511-7.

55. Lei $\mathrm{X}, \mathrm{Xu} \mathrm{XL}$. Cell surface antigen expression of orbital preadipocytes between patients with thyroid-associated ophthalmopathy and healthy adults. Zhong Nan Da Xue Xue Bao Yi Xue Ban. 2008;33(1):43-6.

56. Gillespie EF, et al. Interleukin-6 production in CD40-engaged fibrocytes in thyroid-associated ophthalmopathy: involvement of Akt and NF-kappaB. Invest Ophthalmol Vis Sci. 2012;53(12):7746-53.

57. Hong KM, et al. Differentiation of human circulating fibrocytes as mediated by transforming growth factor-beta and peroxisome proliferator-activated receptor gamma. J Biol Chem. 2007; 282(31):22910-20.

58. Gillespie EF, et al. Increased expression of TSH receptor by fibrocytes in thyroid-associated ophthalmopathy leads to chemokine production. J Clin Endocrinol Metab. 2012;97(5):E740-6.

59. Ko, S.H., et al., The role of stem cells in cutaneous wound healing: what do we really know? Plast Reconstr Surg. 127 Suppl 1: p. 10S$20 \mathrm{~S}$.

60. Gimble JM, Katz AJ, Bunnell BA. Adipose-derived stem cells for regenerative medicine. Circ Res. 2007;100(9):1249-60.

61. Hanson SE, Gutowski KA, Hematti P. Clinical applications of mesenchymal stem cells in soft tissue augmentation. Aesthet Surg J. 2010;30(6):838-42.

62. Lequeux C, et al. Subcutaneous fat tissue engineering using autologous adipose-derived stem cells seeded onto a collagen scaffold. Plast Reconstr Surg. 2012;130(6):1208-17.

63. Renner R, Harth W, Simon JC. Transplantation of chronic wounds with epidermal sheets derived from autologous hair follicles-the Leipzig experience. Int Wound J. 2009;6(3):226-32. 
64. $\mathrm{Lu} \mathrm{F}$, et al. Improved viability of random pattern skin flaps through the use of adipose-derived stem cells. Plast Reconstr Surg. 2008;121(1):50-8.

65. Nambu M, et al. Enhanced healing of mitomycin C-treated wounds in rats using inbred adipose tissue-derived stromal cells within an atelocollagen matrix. Wound Repair Regen. 2007; 15(4):505-10.

66. Nambu M, et al. Accelerated wound healing in healing-impaired $\mathrm{db} / \mathrm{db}$ mice by autologous adipose tissue-derived stromal cells combined with atelocollagen matrix. Ann Plast Surg. 2009;62(3): 317-21.

67. Rigotti, G., et al., Clinical treatment of radiotherapy tissue damage by lipoaspirate transplant: a healing process mediated by adipose-derived adult stem cells. Plast Reconstr Surg, 2007. 119(5): p. 1409-22; discussion 1423-4.

68. Chien $\mathrm{MH}$, et al. Systemic human orbital fat-derived stem/stromal cell transplantation ameliorates acute inflammation in lipopolysaccharide-induced acute lung injury. Crit Care Med. 2012;40(4):1245-53.

69. - Beeson W, Woods E, Agha R. Tissue engineering, regenerative medicine, and rejuvenation in 2010: the role of adipose-derived stem cells. Facial Plast Surg. 2011;27(4):378-87. This article reviews the history of soft tissue augmentation using adipose tissue grafting and the advent of using adipose-derived stem cells. The state-of-the-art stem cell isolation technique as well as anticipated future therapeutic indications are also addressed.

70. Matsumoto D, et al. Cell-assisted lipotransfer: supportive use of human adipose-derived cells for soft tissue augmentation with lipoinjection. Tissue Eng. 2006;12(12):3375-82.
71. - Yoshimura K, et al. Cell-assisted lipotransfer for facial lipoatrophy: efficacy of clinical use of adipose-derived stem cells. Dermatol Surg. 2008;34(9):1178-85. This study evaluated the efficacy and adverse effects of lipoinjection and supplementation of adipose-derived stem/stromal cells (ASCs) to adipose grafts using a novel strategy called cell-assisted lipotransfer (CAL). In CAL, stromal vascular fraction containing ASCs was freshly isolated from half of an aspirated fat sample and attached to the other half of aspirated fat sample with the fat acting as a scaffold. Their results suggest that CAL is both effective and safe and potentially superior to conventional lipoinjection for facial recontouring.

72. $\mathrm{Wu} \mathrm{L}$, et al. Secreted factors from adipose tissue increase adipogenic differentiation of mesenchymal stem cells. Cell Prolif. 2012;45(4):311-9.

73. HODGEKISS, A., 21 December 2012.

74. Park BS, et al. Adipose-derived stem cells and their secretory factors as a promising therapy for skin aging. Dermatol Surg. 2008;34(10):1323-6.

75. Kim WS, Park BS, Sung JH. Protective role of adipose-derived stem cells and their soluble factors in photoaging. Arch Dermatol Res. 2009;301(5):329-36.

76. Mojallal A, et al. Improvement of skin quality after fat grafting: clinical observation and an animal study. Plast Reconstr Surg. 2009;124(3):765-74.

77. Oni, G., et al., Transdermal delivery of adipocyte-derived stem cells using a fractional ablative laser. Aesthet Surg J. 33(1): 109-16.

78. Baek RM, et al. The effect of botulinum toxin A on fat graft survival. Aesthetic Plast Surg. 2012;36(3):680-6. 